\title{
Developments in Non-Fire Sensitivity Testing of Optical Smoke Detectors - Proposal for a New Test Method
}

\author{
WOLFGANG KRÜLL ${ }^{1}$, THORSTEN SCHULTZE ${ }^{1}$, INGOLF WILLMS ${ }^{1}$, and ANDRÉ FREILING ${ }^{2}$ \\ ${ }^{1}$ Department of Communication Systems (NTS) \\ University of Duisburg-Essen, Germany \\ ${ }^{2}$ Airbus Operations $\mathrm{GmbH}$ \\ Bremen, Germany
}

\begin{abstract}
The purpose of an automatic fire detection system is the fast and reliable detection of arising fires in order to keep damage as low as possible. The European Standard EN54 defines a series of tests to prove and certify the functionality of smoke detectors, i.e. to prove that the detector is able to detect a fire in a prescribed period of time. Unfortunately the complex problem of avoiding false alarms is not completely addressed. In contrast to the well standardised methods for the evaluation of the detection capability of a smoke detector, there is a lack of a reproducible and representative test method concerning the false alarm susceptibility with regard to nuisance aerosols. The consequences of false alarms should not be underestimated, as they may cause several costs to the operator, especially in airborne applications.

Several approaches are possible to reduce the false alarm susceptibility of optical smoke detectors, e.g. different wavelengths and scattering angles. Unfortunately the developer has no test methods to quantify improvements due to new developments. This paper presents a new approach for the test of smoke detectors regarding their susceptibility to false alarms due to nuisance aerosols, like steam and dust. Fog and spray tests in the developed set-up are planned. The presented test apparatus is a helpful tool for developers as well as for test houses during the developing and certification process. System designer will have a decision criterion to find an adequate detector for a specific place of installation.

The objective of the project was the development of a standardised test method for sensitivity tests of fire detectors to hazardous substances. The presented test apparatus has been developed in a cooperation of Airbus and the University of Duisburg-Essen and can contribute to a test standard. The design is similar to the EN54 test duct, however due to its small volume of only around 321 , the duct is much smaller, easier to clean, portable and cheaper. The duct allows a qualitative statement on the sensitivity of the tested detector regarding nuisance aerosols with reproducible results. Also a slow pollution with dust of the measuring chamber can be emulated. The achieved results of this study are a promising first step in the development of a new test standard.
\end{abstract}

KEYWORDS: test apparatus, test method, false alarms, non-fire sensitivity, smoke detectors, dust.

\section{INTRODUCTION}

In many applications a fire will first develop as a smouldering fire, often producing smoke and combustion gases over hours before a visible flame arises. Thus, commonly smoke detectors are implemented, which are able to detect small concentrations of smoke in the monitored atmosphere.

However, during its 'fire-free' lifetime a smoke detector is not only exposed to clean air. Dust, steam, fog and other aerosols may pollute the monitored air and, if the detector is not able to distinguish them from smoke, cause a false alarm. False alarms can be very costly, as they may e.g. cause the interruption of the production line or force a pilot to an emergency landing at the next airport.

In 2008, a total number of 374 fire alarm systems were connected to the Duisburg Fire Brigade. 533 alarms coming from automatic systems were registered [1]. The pie chart in Fig. 1 shows the ascertained reasons for the alarm. Only $16 \%$ of the alarms were triggered by a real fire. Further on, it is noteworthy that in $44 \%$ of the cases, the detector indeed worked correctly and activated an alarm due to a monitored aerosol, which however was not smoke. Hence the implemented detectors were not able to distinguish smoke from other aerosols. This shows that the problem of false alarms should not be underestimated.

In airborne applications this situation is even worse. Due to the high safety standards in aviation, a fire in the cargo compartment of an airplane, emulated by a test apparatus with a paraffin smoke generator, has to 
be detected within only $60 \mathrm{~s}$. This leads to highly sensitive detectors with low alarm thresholds, but it unfortunately also implicates a high number of false alarms. Actually the false alarm rate is about 100:1, i.e. out of 100 alarms, only one is a real fire [2].

To overcome this major problem in the recent years big efforts have been made in order to reduce the susceptibility of smoke detectors to nuisance aerosols. A good overview of the different approaches and the actual state of the art is given in the proceedings of the $14^{\text {th }}$ International Conference on Automatic Fire Detection, AUBE '09 [3].

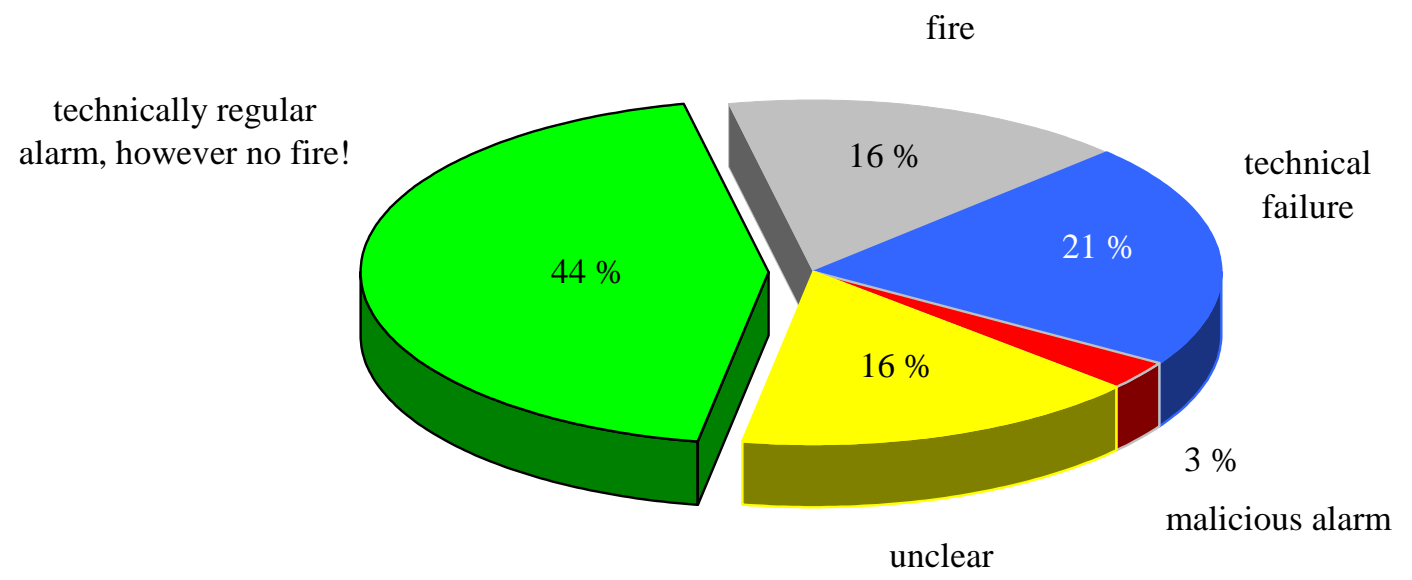

Fig. 1. Causes of automatic fire alarms in Duisburg, 2008.

However, for system designers which integrate these new generation detectors, the problem of comparing and qualifying the different technologies arises. In contrast to the well standardised methods for the evaluation of the detection capability of a fire detector, there is a lack of reproducible and representative test methods concerning the false alarm susceptibility.

This paper presents the recent work on a test method for determining the sensitivity of smoke detectors to non-fire aerosols. The method has been developed in a cooperation of Airbus and the University of Duisburg-Essen and can contribute to a test standard. Focus of the test is the susceptibility of smoke detectors to dust, the major source of false alarms in airborne applications. Testing with steam, fog, sprays and other aerosols are planned in order to evaluate the detector in other possible non-fire scenarios.

\section{SOURCES AND PROPERTIES OF DUST}

Designing a new test method for non-fire sensitivity testing of smoke detectors requires adequate knowledge of dust properties. Due to the influence on health, the impact of dust within the climate and global ecological system is of particular importance. Thus, an increasing interest in the environmental factor dust became of essential significance. New methods of measurement allow characterizing dust and its fluctuation with highest accuracy, leading to the knowledge that larger particles (larger than $100 \mu \mathrm{m}$ ) including room dust, coarse sand and soot aggregates fall out quickly. Medium-size particles in the range $1 \mu \mathrm{m}$ to $100 \mu \mathrm{m}$ settle out slowly (e.g. pollen, fly ash, coal dust, fine sand). Small particles (less than $1 \mu \mathrm{m}$, e.g. soot and tobacco smoke) fall slowly, in a quiet atmosphere, it takes days up to years for dust to settle out and it can be carried more than $1000 \mathrm{~km}$, but it can be washed out by rain. Beside space-based dust (40,000 tonnes each year [4]) two significant sources of dust are:

- Man-made sources e.g. soot, agriculture, housekeeping, rubber scrubbed from vehicle tires, power plants and heating installations, industrial processes, transport exhaust.

- Natural sources e.g. volcanic eruptions, soil erosion, sea salt, combustion of biomass, forest and grassland fires, dust storms, pollen.

Aerosols can be classified depending on their size [5]. PM10 (particulate matter) is understood to be the total volume of particles with an aerodynamic diameter $\leq 10 \mu \mathrm{m}$. These particles will pass through the nose, reaching the lungs. 
Aerosols, such as dust are solid or liquid particles. The shape of the dust particles can be very complex with sharp edges and rough surfaces compared with a spherical shape. Figure 2 shows the particle size range of some aerosols with environmental relevance. It can be seen that the particle size of some nuisance aerosols are close to the particle size of smoke caused by a fire. Hence common smoke detectors are not able to distinguish a real fire from a non-fire situation.

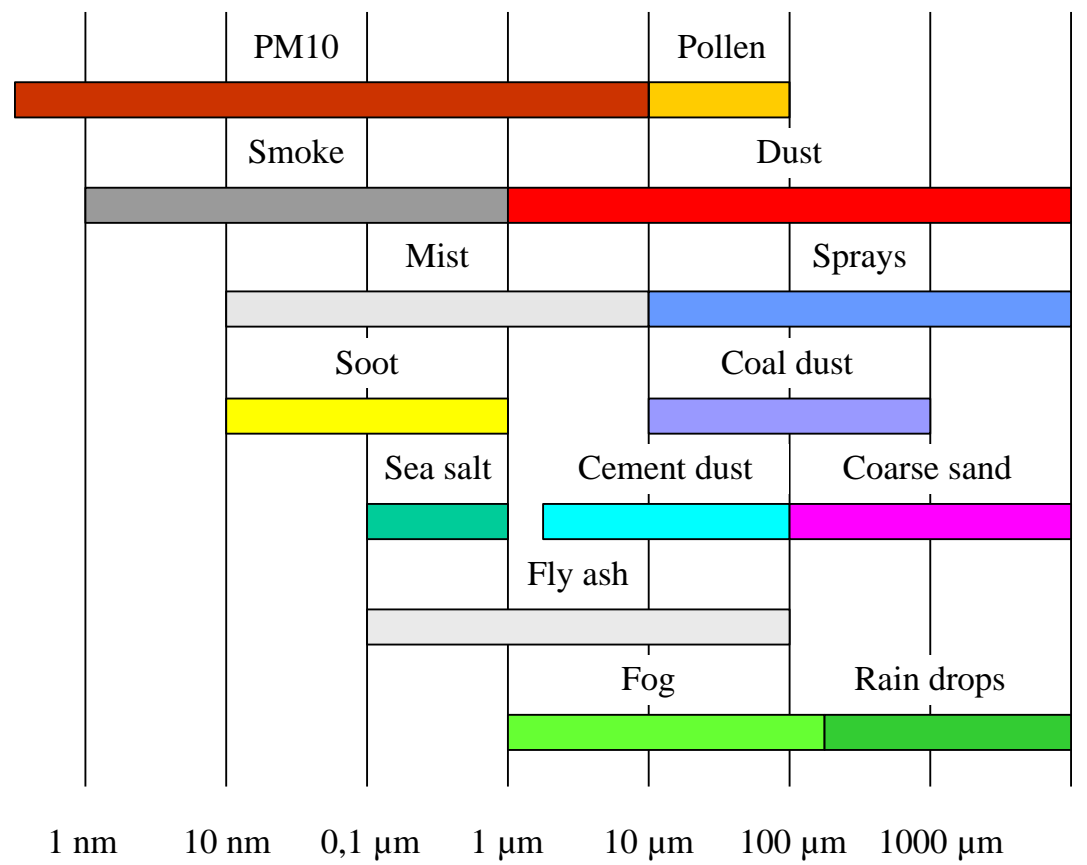

Fig. 2. Example for the particle size of aerosols with environmental relevance [6].

The constructional design of an optical detector, e.g. its wavelength and scattering angle, has a large effect on the false alarm susceptibility of smoke detectors. Thus, different approaches to reduce the false alarm rate exist, however there is a lack of qualitative and quantitative comparison between the methods. The presented new duct-type test chamber shall achieve two main aims. On one side it is a tool for the manufacturer of smoke detectors to minimize the false alarm susceptibility and on another side it can be an important tool during the certification process.

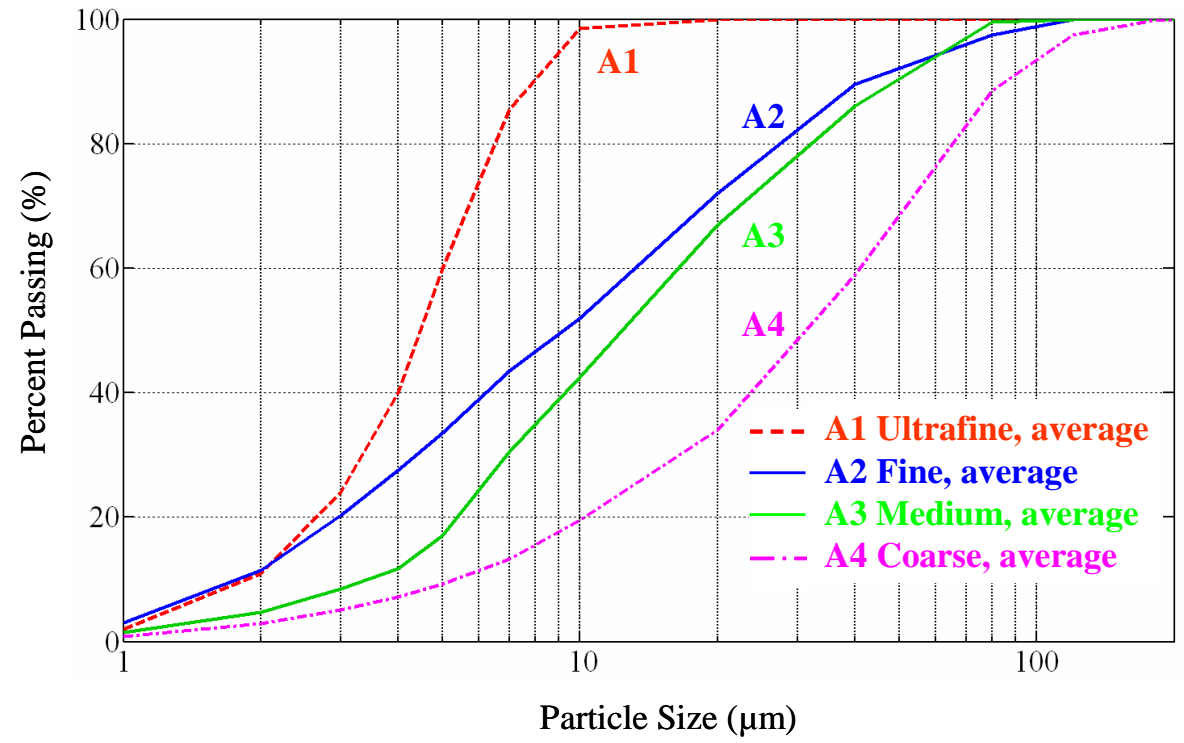

Fig. 3. Graphical comparison of ISO 12103-1 test dust grades [7]. 
Four grades of test dust including dust description, designation, particle size distribution, particle size analysis procedure, and chemical content [8] are specified. Figure 3 shows the graphical comparison of ISO test dust grades based on ISO 12103-1 specified limits (cumulative volume):

- A1 dust Ultra fine, dust particles with nominal 0-10 $\mu \mathrm{m}$ size.

- A2 dust Fine, dust particles with nominal 0-80 $\mu \mathrm{m}$ size.

- A3 dust Medium test dust with nominal 0-80 $\mu \mathrm{m}$ size with a lower $0-5 \mu \mathrm{m}$ content than A2.

- A4 dust Coarse test dust with nominal 0-180 $\mu \mathrm{m}$ size.

The intent in producing a separate standard for the description of test dust was to provide a simple and accurate reference for use in other standards or documents where test dust use is required [7]. In order to avoid health hazardous substances such as quartz, DMT dolomite mineral is used. This quartz-free material has a standardised grain size distribution [9]. Figure 4 shows the volume fraction of the DMT Dolomite 10 and Dolomite 90 test dust. Dolomite is a vacuum cleaner test dust in accordance with DIN IEC 60312.

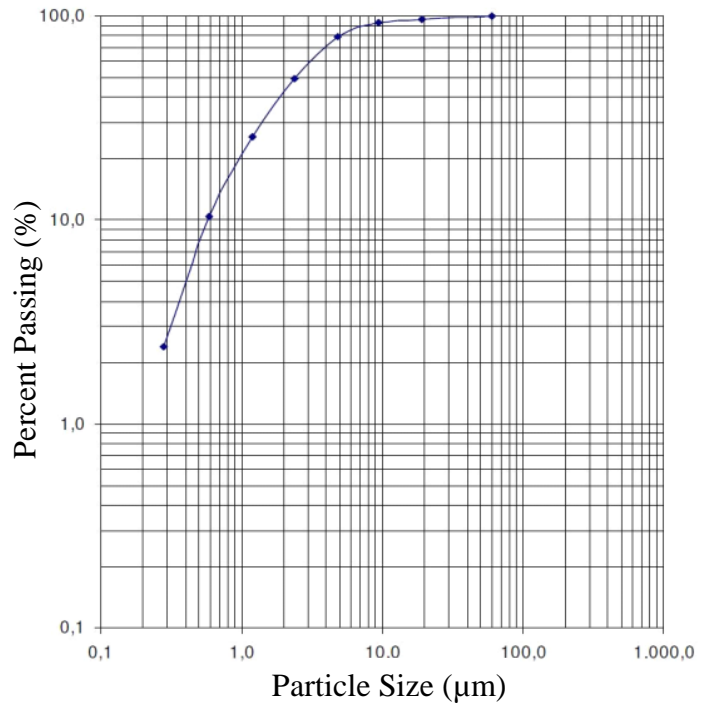

(a)

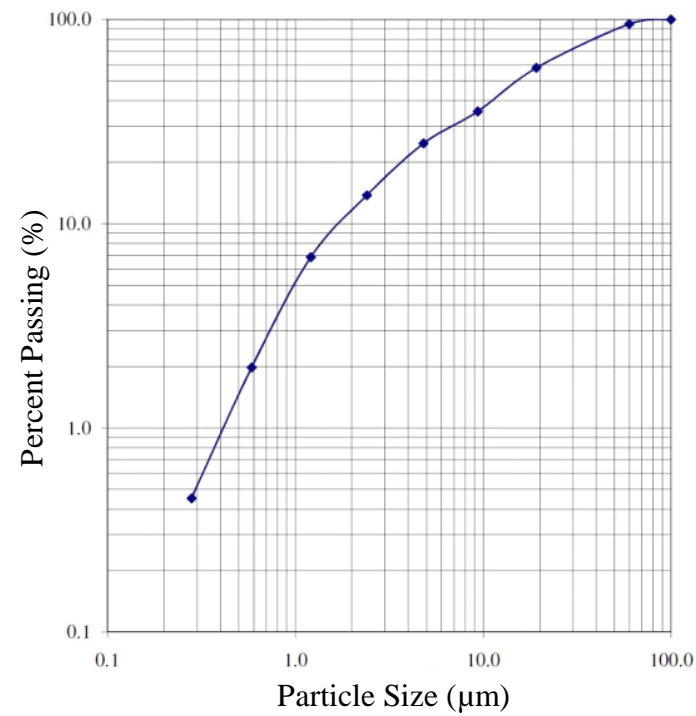

(b)

Fig. 4. Volume fraction (\%): (a) Dolomite 10 test dust; (b) Dolomite 90 test dust [9].

\section{FALSE ALARM REJECTION TEST CHAMBER}

The first non-fire tests were carried out in the EN54 smoke duct. A big drawback is the large volume, thus a significant amount of dust is required for usable dust concentrations. Dust particles are heavy compared to paraffin oil used for EN54 tests, so a fast deposit can be seen. Cleaning the EN54 smoke duct after such a dust test is very time-consuming. Therefore it was necessary to develop a new test chamber. Siemens Aerospace (France) has set up such a test chamber in the context of the development of the multi-criteria smoke detector used in Airbus A380 in order to demonstrate its false alarm rejection capabilities [11]. Figure 5 shows the EN54 smoke duct as well as the test chamber used by University of Duisburg-Essen which follows the design idea of the Siemens test set-up to some degree. A lot of tests were performed to evaluate the applicability as well as the functionality.

The test chamber consists of a hemispherical plastic / carbon hood with an attached cylinder. Figure 6 shows the principle design. Feeding such dust types manually into a smoke test channel typically leads to a hardly reproducible particle density distribution as many particles stick to each other giving a higher density of larger particles. For this reason the Palas RBG 1000 powder disperser for extremely low and medium mass flow was used in addition to the Siemens Aerospace design. A special advantage of the system is a quick and easy exchange of the powder reservoir as well as changing the mass flow. In Fig. 7 the principle of the powder disperser is shown. The powder has to be filled into a cylindrical reservoir and 
is transported onto a rotating brush. Dispersing air streams over the brush and pulls the powder out of the brush [10]. Dosing is performed using the precisely controlled feed rate of the feed piston.
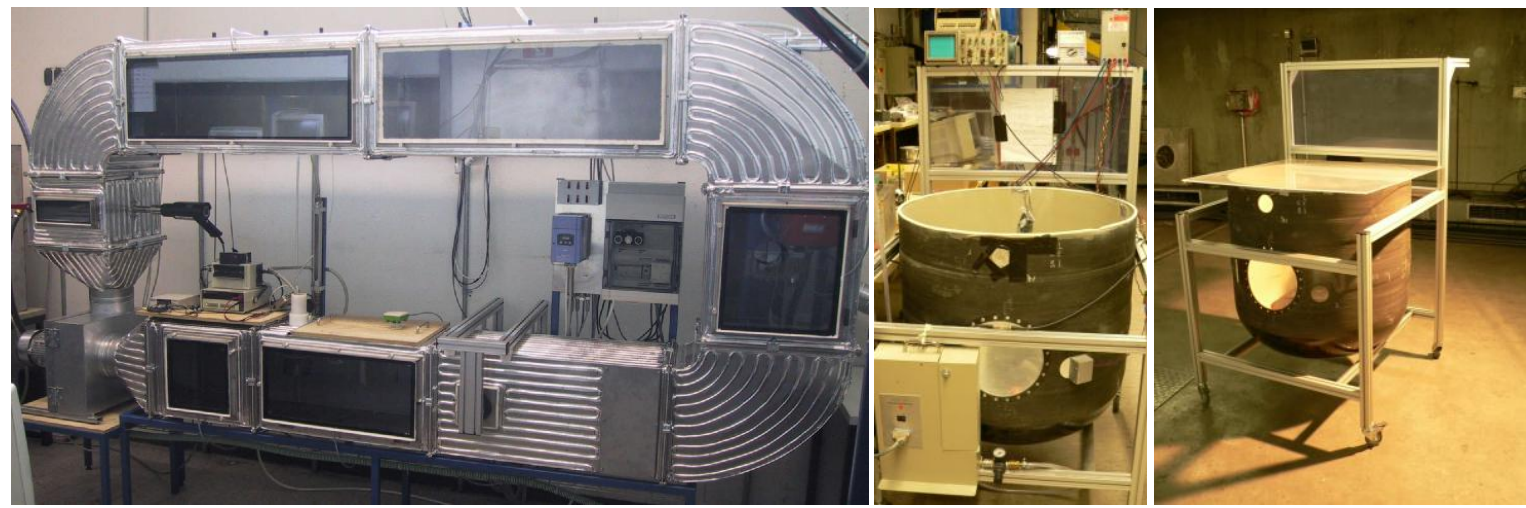

Fig. 5. EN54 smoke duct and false alarm rejection test chamber.
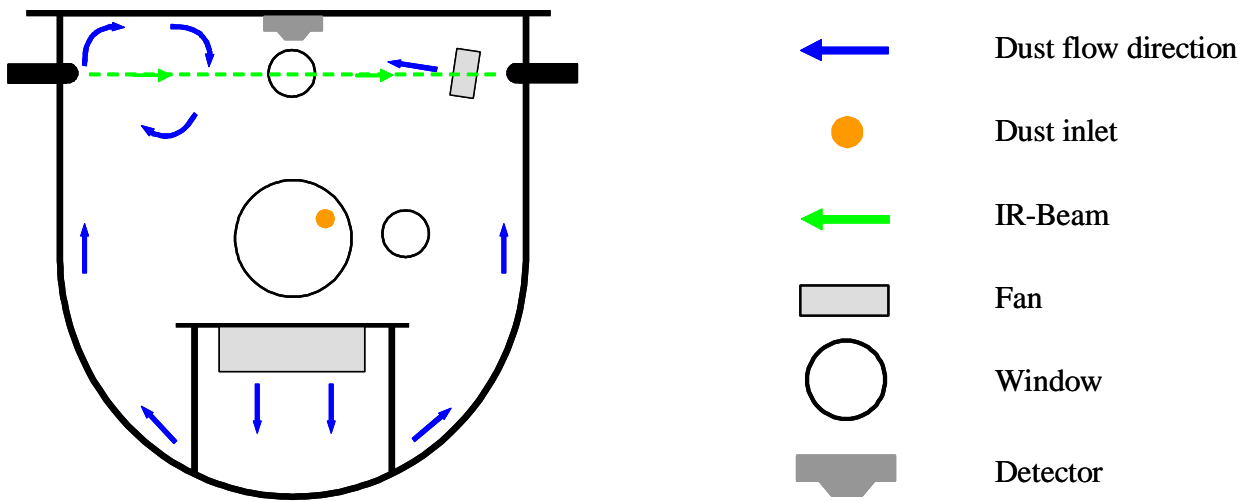

Fig. 6. Schematic of the false alarm rejection test chamber.

The following parameters are used for control of the aerosol concentration:

- Diameter of the reservoir

- Feed rate

- Dispersing air pressure

- Rotating speed of the brush

- Dust type
$7 \mathrm{~mm}, 14 \mathrm{~mm}, 28 \mathrm{~mm}$

$1 \mathrm{~mm} / \mathrm{h}$ to $590 \mathrm{~mm} / \mathrm{h}$

0.5 bar to 2 bar

$600 \mathrm{rpm}$ to $1200 \mathrm{rpm}$

DMT-Dolomite dust $<90 \mu \mathrm{m}$

aerosol

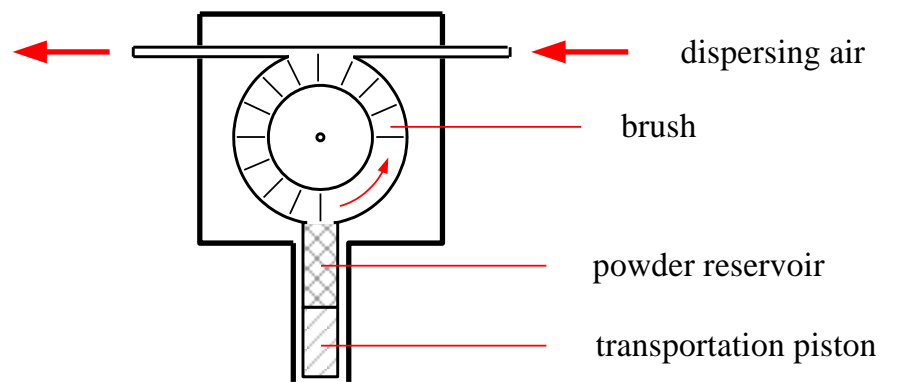

feed

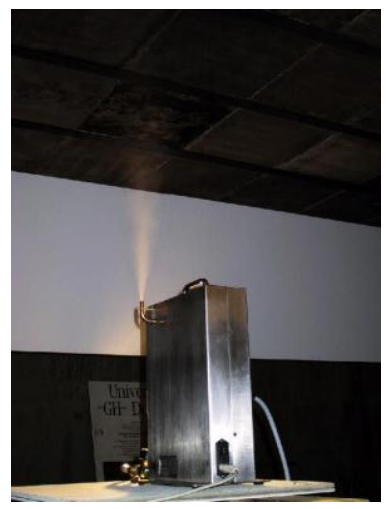

Fig. 7. Palas powder disperser RBG 1000. 
A large fan on the base of the chamber and a second fan at the top cause a rotating air flow. An obvious disadvantage of this chamber is an unrealistic highly turbulent flow around the detector. The situation in an EN54 test duct with an almost homogenous flow velocity within a square cross-section is a much better approximation of reality. Moreover it is not possible to define the directionality of a smoke detector against dust. Air flow was measured at different positions below the top of the chamber as well as close to the side walls. The biggest turbulences were measured at the detectors' position (see Fig. 8). The light extinction was measured about $100 \mathrm{~mm}$ below the top of the chamber. The transmitter and receiver were mounted in the side walls. Unfortunately, the results of the performed tests have not been particularly satisfactory. The measured light obscuration (in $\% / \mathrm{m}$ ) and the extinction (in $\mathrm{dB} / \mathrm{m}$ ) of two tests with same parameters of dust production are shown in Fig. 9. The result is a linear rise of the dust concentration; however the slope is to some degree unpredictable and not adjustable. Therefore the reproducibility is not guaranteed.

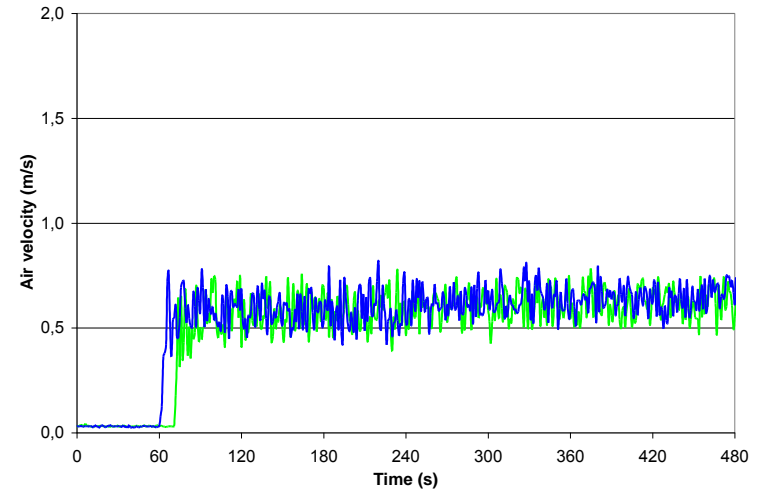

(a)

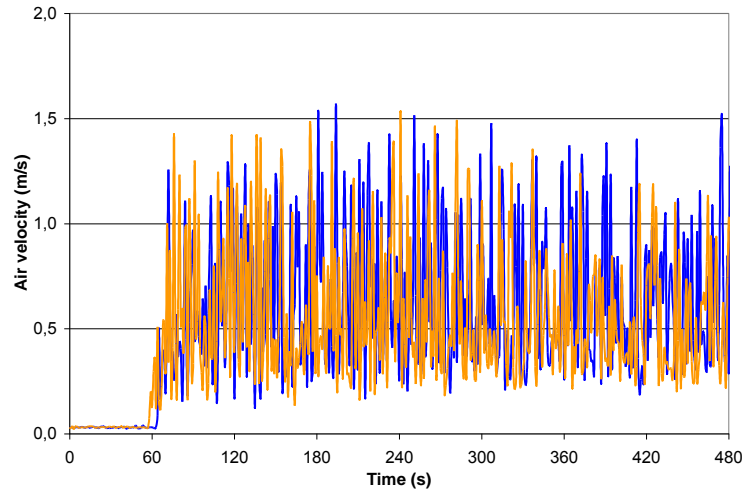

(b)

Fig. 8. Air flow at different positions inside the false alarm rejection test chamber: (a) air flow close to the side walls; (b) air flow at the detectors' position.

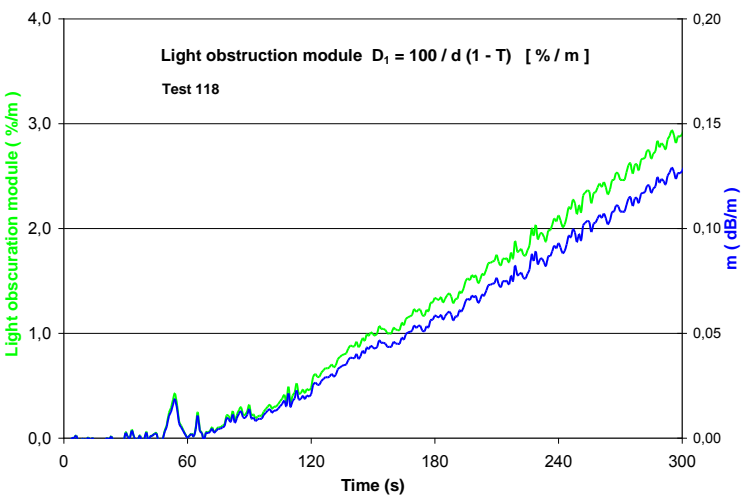

(a)

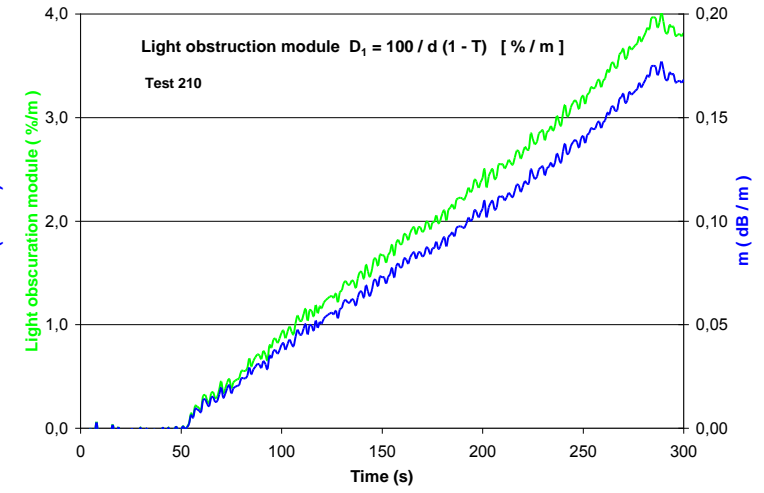

(b)

Fig. 9. Examples of dust concentration inside the false alarm rejection test chamber: (a) measurement 1;

(b) measurement 2 .

\section{DUCT TYPE TEST CHAMBER}

In order to avoid strong fluctuations of the flow velocity and flow direction, a second set-up has been developed in the form of a closed duct with rectangular profile similar to the EN54 test duct. The duct consists of two concentric $150 \mathrm{~mm}$ high stainless steel rings, with a diameter of $300 \mathrm{~mm}$ and $600 \mathrm{~mm}$ respectively. The ceiling and floor of the duct are closed by two plastic plates, which are coated with stainless steel. The achieved cross-section of the duct is $150 \mathrm{~mm} \times 150 \mathrm{~mm}$, with a mean length of about $1.4 \mathrm{~m}$. Figs. 10 to 13 outline the developed duct. In Fig. 10 the detector is shown in a position on the duct's 
base for a better overview of the different components inside the duct. In the new chamber, the detector is mounted at the duct ceiling, as required by the manufacturer.

The chosen set-up shows several advantages over the EN54 duct and the false alarm rejection test chamber:

- The compact design of the new test duct provides a portable device with little weight.

- The duct is easy to clean because of the very small volume (about $32 \mathrm{l}$ ) and the smooth surface.

- A widely laminar air flow with adjustable tip velocity and flow direction is given.

- Controlled and reproducible dust dispersion is possible.

- The low volume allows the reduction of the used aerosol quantity.

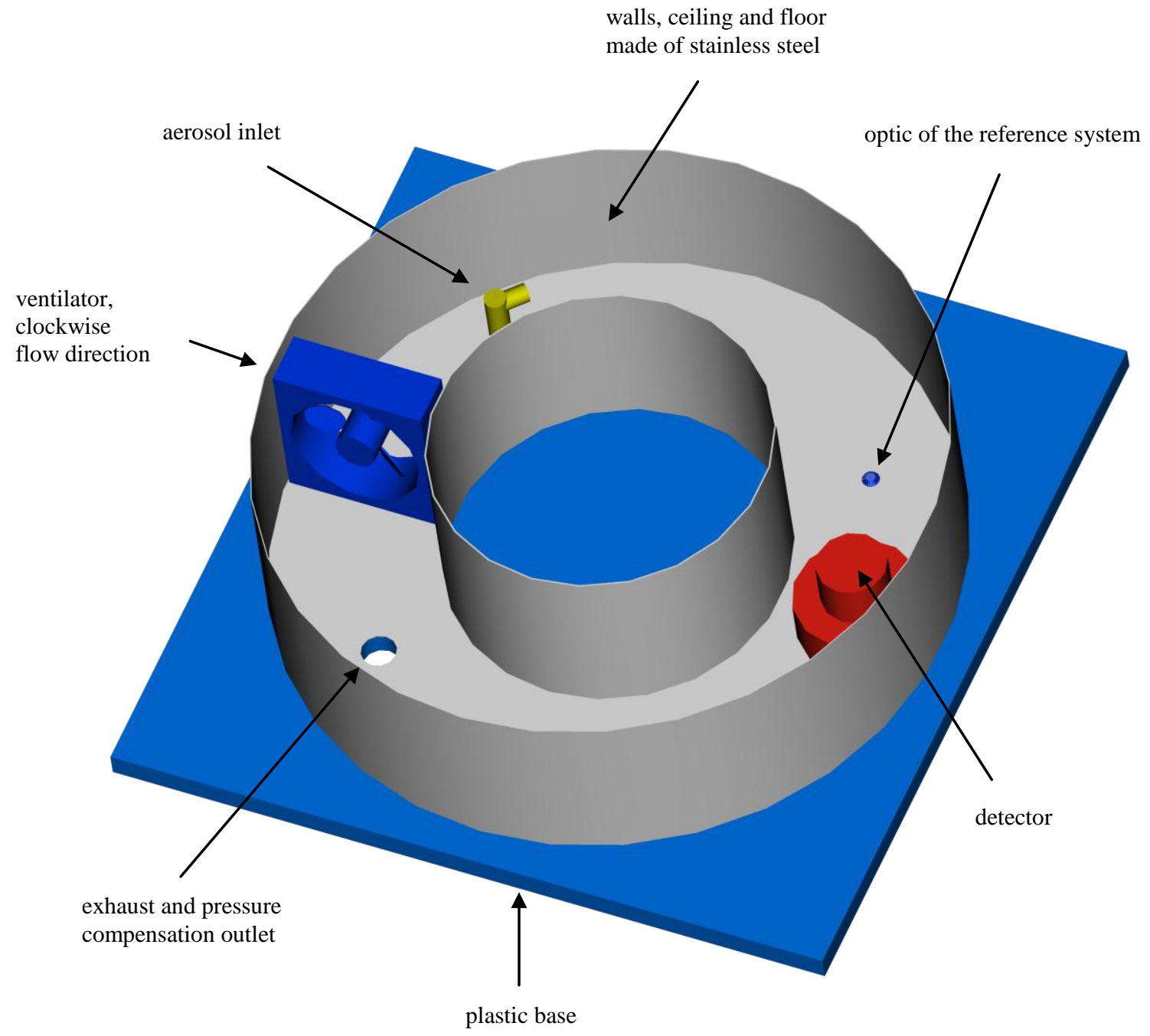

Fig. 10. Outline of the developed test duct.

Due to the highly conductive walls and the small volume of the duct, also tests with hazardous or combustible aerosols are possible. The airflow in the duct is generated by an encapsulated motor with a mounted airscrew giving a flow velocity between $0.2 \mathrm{~m} / \mathrm{s}$ and $1 \mathrm{~m} / \mathrm{s}$ as required by the EN54 [12] for the test of detectors when using atomised paraffin oil. The flow velocity was measured by the hot-wire anemometer EE70-VT315 [13]. 


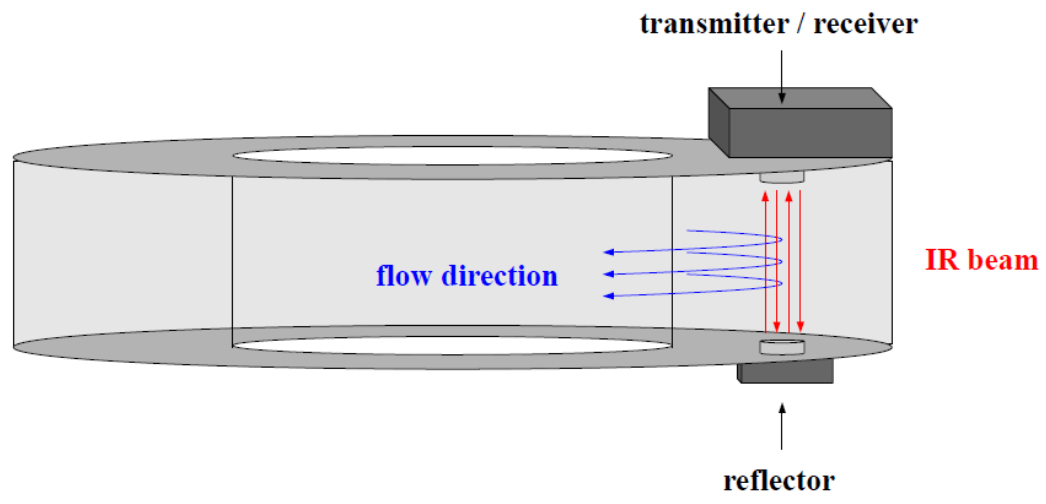

Fig. 11. Sketch of the reference extinction path.

Therefore the test scenario of the detector in the non-fire case is similar to functional testing according to EN54. To ensure a better comparison of the achieved results with these tests, the reference system implemented in the duct works as defined in EN54, i.e. according to the light extinction measurement. EN54 prescribes a light extinction measurement in the near-IR range for the test of smoke detectors based on the light scattering principle. The evaluated measurement parameter is the amount of light loss (due to extinction) normalised by the measurement path, i.e. the extinction module expressed in $\mathrm{dB} / \mathrm{m}$ and the light obscuration in $\% / \mathrm{m}$. However, the extinction path in the new duct is about 2.5 times shorter than the path in the EN54 duct or the test chamber of Fig. 6. Due to the small cross-section and the need for very precise extinction measurement, the extinction measuring device Lorenz AML [13] had to be adapted. The AML is an approved reference measurement system for EN54 tests and consists of a transmitter / receiver module and a reflector. The channel only requires two opposite-facing windows with a diameter of at least $65 \mathrm{~mm}$. Figure 11 depicts the position of the reference extinction path in the duct.
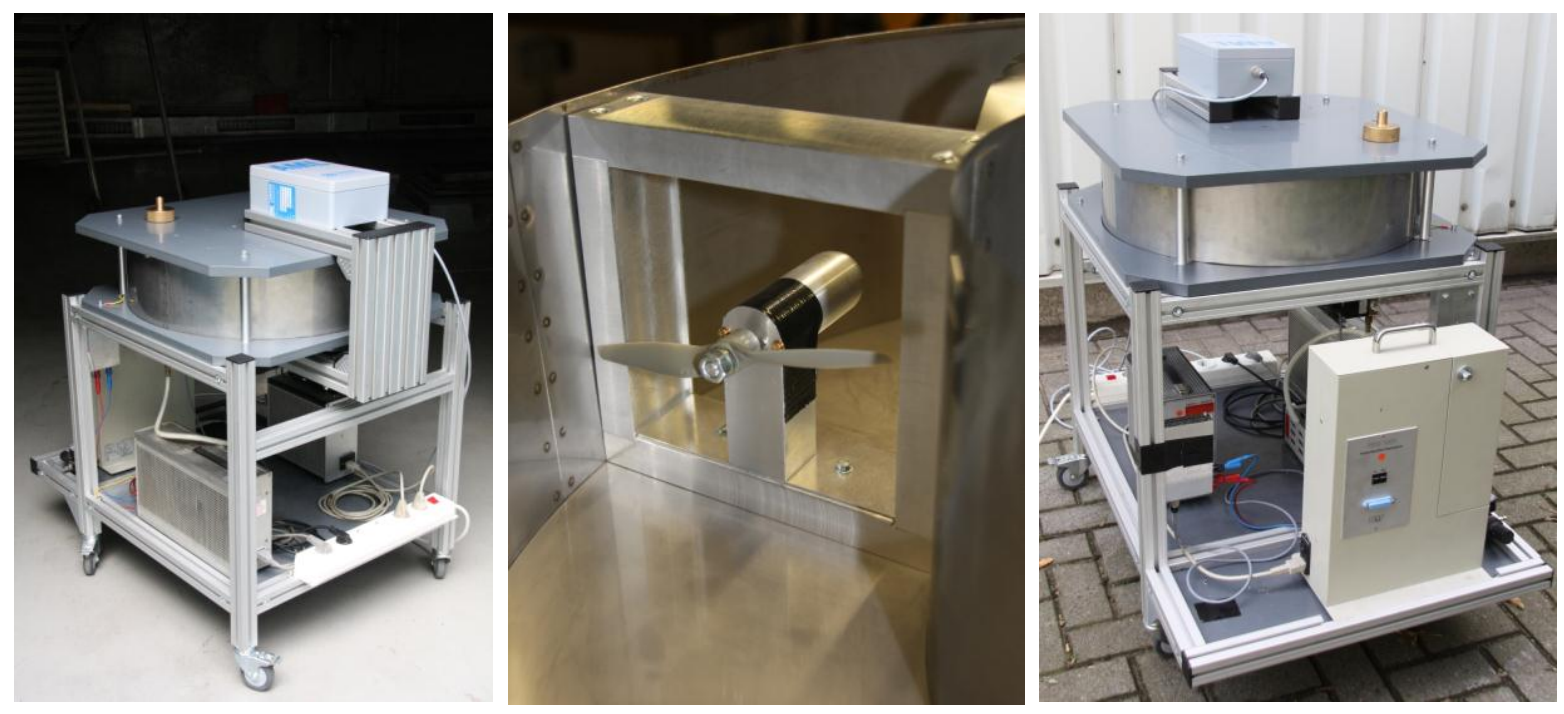

Fig. 12. Different views of the developed test duct.

Due to the small volume, another deciding factor was the development of a reproducible and in a wide range adjustable slow and precise aerosol feeding. The already precise control of the aerosol generator had to be extended by an adjustable chopper placed just before the aerosol inlet which reduces the amount of dust entering the duct. The chopper is controlled by a PC and therefore different slopes of the concentration can be preset. Due to the high chopping frequency and the low-pass properties of the duct the slopes are still linear. 

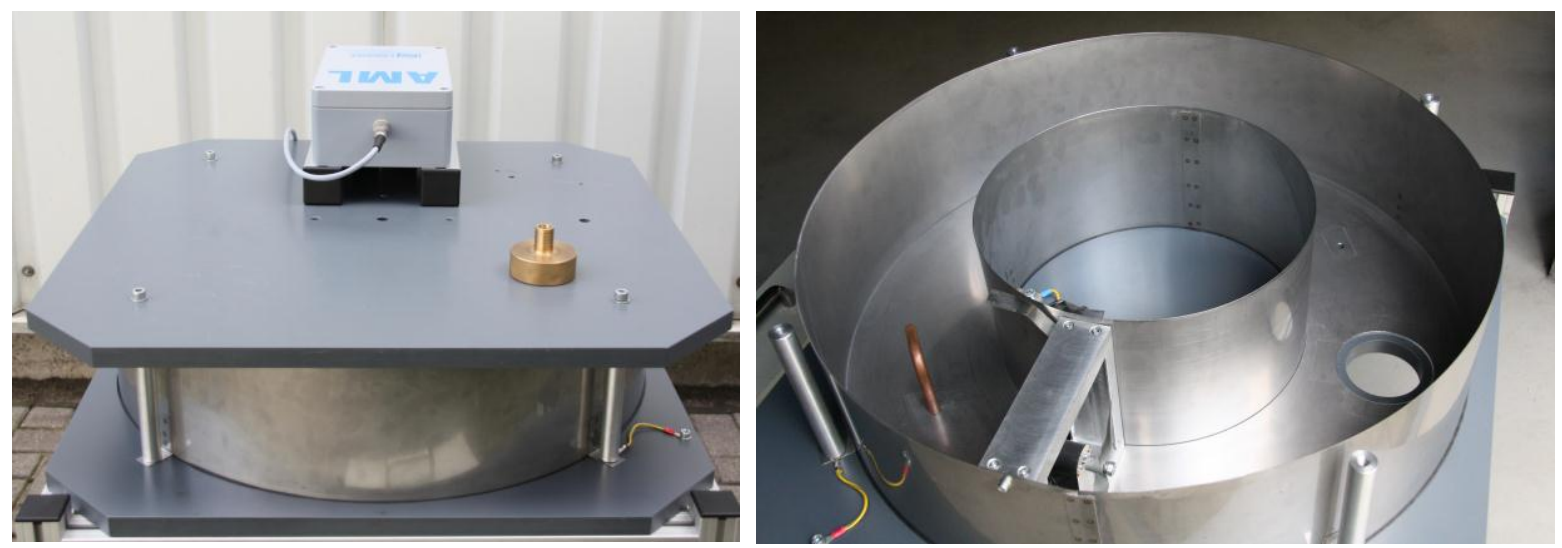

Fig. 13. Different views of the developed test duct.

\section{MEASUREMENT RESULTS}

Several tests with dolomite sand $<90 \mu \mathrm{m}$ were performed, varying all parameters of the Palas RBG 1000 powder disperser to realise a slow and linear increase of dust concentration over time. To perform dust tests, solid cleaning of all devices at the beginning is required; otherwise, it is possible to have a large dust cloud when starting the test procedure causing biased results.

Following EN54 tests measuring the directionality or the response behaviour of a smoke detector the increase of the aerosol concentration has to be within the following limits [12]:

$$
0.015 \leq \frac{\Delta \mathrm{m}}{\Delta \mathrm{t}} \leq 0.1\left(\mathrm{~dB} \mathrm{~m}^{-1} \cdot \mathrm{min}^{-1}\right)
$$

The slew rate of the smoke density in the EN54 test duct typically is set to be about $0.06 \mathrm{~dB} \mathrm{~m}^{-1} \mathrm{~min}^{-1}$. In the following figures (Figs. 14 and 15) it is shown that a slow and very linear increase of the dust concentration can be achieved at different rates of rise by chopping the dust flow by an electromagnetic valve. Each figure shows several test results. The schematic of the chopped dust production is shown in Fig. 16a. The behaviour of the dust concentration can be set by varying the cycle time and duration. The achieved linearity is a precondition for performing dust tests in a similar manner as smoke tests according to EN54. The visualisation and data conversion was done by LabView-based software.

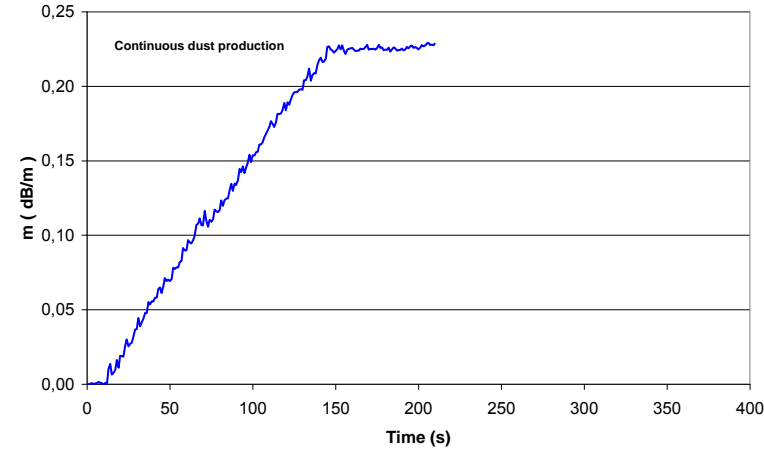

(a)

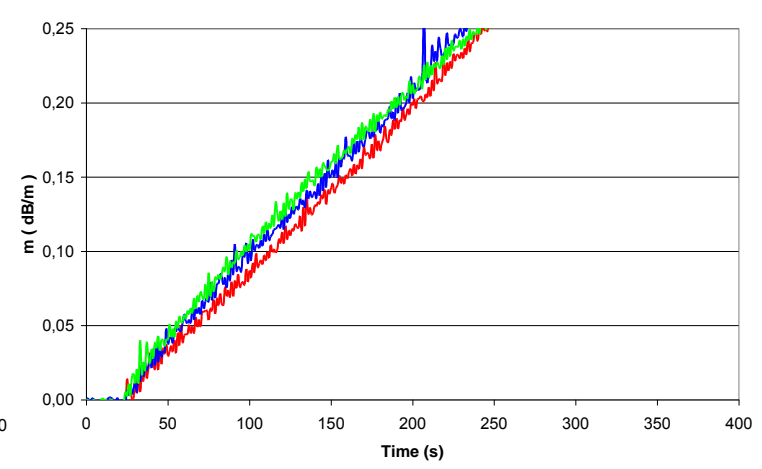

(b)

Fig. 14. Examples for the dust production in the new test duct: (a) continuous dust production; (b) chopped dust production, slew rate $\approx 0.06 \mathrm{~dB} \mathrm{~m} \cdot \mathrm{min}^{-1}$.

Several optical smoke detectors have been tested. Some of them had no alarm up to a dust concentration of $2 \mathrm{~dB} / \mathrm{m}$, others had an alarm very early. An example of the chopped dust production and alarm times of one of the tested detectors is shown in Fig. 16b. Both tests were done with different slew rates 
$\left(\approx 0.05 \mathrm{~dB} \mathrm{~m}^{-1} \cdot \min ^{-1}\right.$ and $\left.0.08 \mathrm{~dB} \mathrm{~m} \mathrm{~m}^{-1} \cdot \mathrm{min}^{-1}\right)$. It can be seen that the alarm of the detector depends on the measured dust concentration.

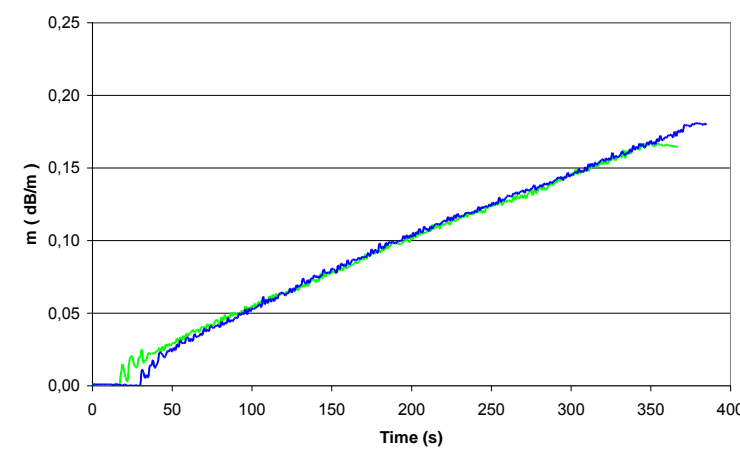

(a)

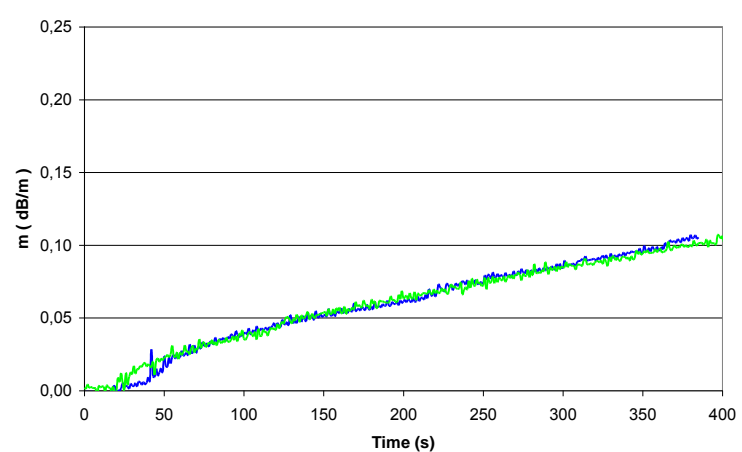

(b)

Fig. 15. Chopped dust production: (a) slew rate $\approx 0.032 \mathrm{~dB} \mathrm{~m} \cdot \mathrm{min}^{-1}$; (b) slew rate $\approx 0.016 \mathrm{~dB} \mathrm{~m}^{-1} \cdot \mathrm{min}^{-1}$.

After testing several optical smoke detectors the system designer will have a decision criterion to find an adequate detector for a specific place of installation.

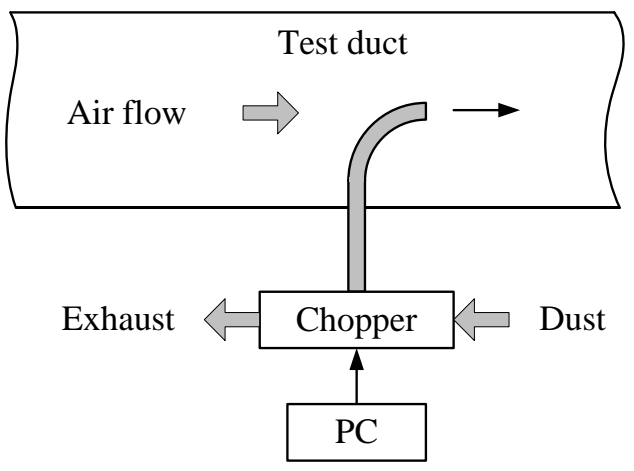

(a)

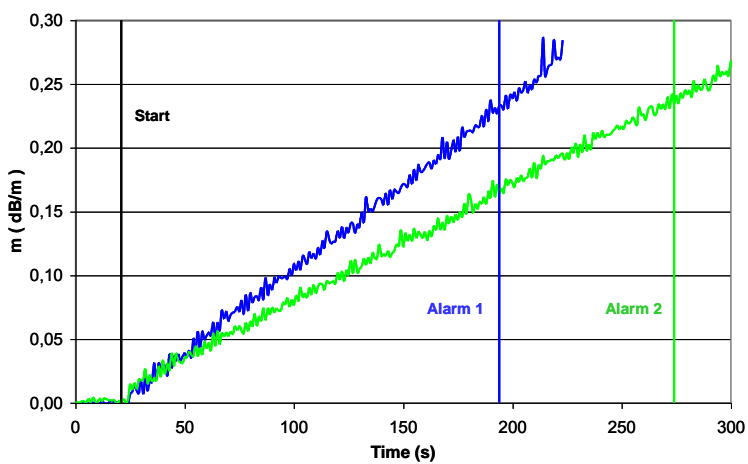

(b)

Fig. 16. Chopped dust production: (a) schematic of the chopped dust production; (b) alarm times of an optical smoke detector.

In airborne applications many false alarms are caused by cigarette smoke, especially in the lavatories. That was the reason for implementing tests with cigarette smoke in addition to dust tests. Smoke suctioning was controlled by a chopped pump; the schematic is shown in Fig. 17a. The PC-controlled chopper enables reproducible smoke concentrations. The implemented slew rate of the smoke density with $\approx 0.06 \mathrm{~dB} \mathrm{~m}{ }^{-1} \cdot \mathrm{min}^{-1}$ fits EN54 requirements (Eq. 1). Again, several optical smoke detectors have been tested. All detectors had an alarm. The measured smoke density of 10 tests and the corresponding alarm times of an optical smoke detector are shown in Fig. 17b. 


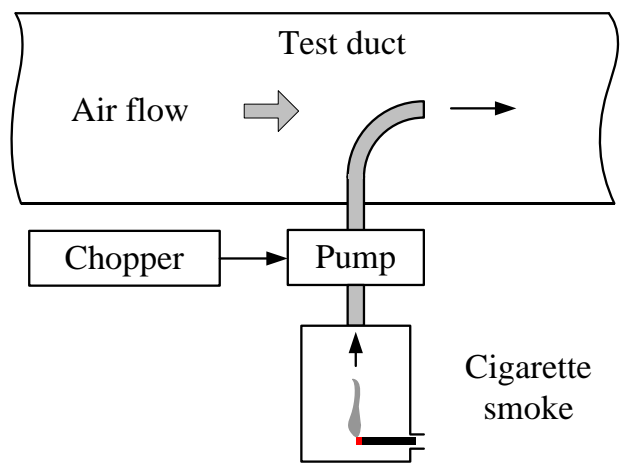

(a)

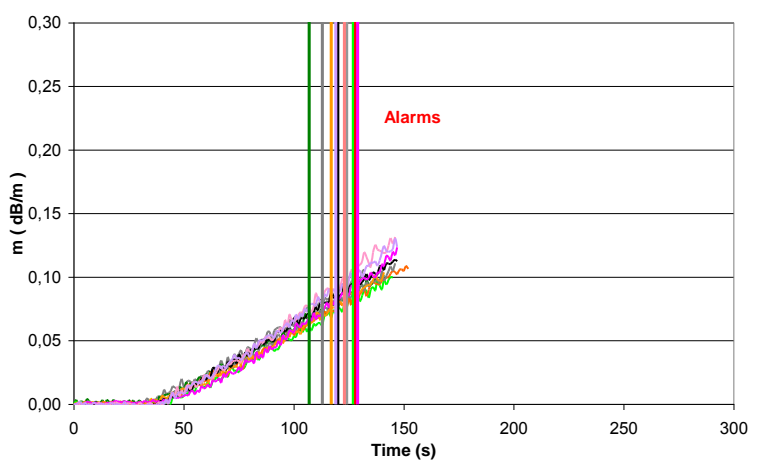

(b)

Fig. 17. Chopped cigarette smoke production: (a) schematic of the chopped cigarette smoke production; (b) chopped cigarette smoke production, and alarm times of an optical smoke detector.

\section{CONCLUSION AND OUTLOOK}

In contrast to the well standardised methods for the evaluation of the detection capability of a fire detector, there is a lack of a reproducible and representative test method concerning the false alarm susceptibility. A first step to fill this gap was done by the development of the false alarm rejection test chamber. The new duct type test chamber is a promising first step for the development of a new test method. Tests have shown sufficiently linear and reproducible data. Extensive measurements are required to define special slew rates for a test standard with dust as aerosol. Testing with steam, fog, sprays and other aerosols are planned in order to evaluate the detector in other possible non-fire scenarios.

\section{REFERENCES}

[1] Feuerwehr Duisburg, Statistics 2008, Duisburg, Germany, 2009.

[2] Blake, D., Aircraft Cargo Compartment Fire Detection, International Aircraft Systems Fire Protection Working Group Atlantic City, NJ, October 30-31, 2002.

[3] Luck, H., and Willms, I., Proceedings of the $14^{\text {th }}$ International Conference on Automatic Fire Detection, AUBE '09, Duisburg, Germany, September 8-10, 2009, ISBN 978-3-940402-01-1.

[4] Cole, M., Aerosol characterisation for reliable ASD operation, $14^{\text {th }}$ International Conference on Automatic Fire Detection, AUBE '09, Duisburg, Germany, September 8-10, 2009.

[5] Pesch, M., Kontinuierliche Messung von Partikelgrößenverteilungen (wide range) am Hohenpeißenberg, Seminar "Nanopartikel in der Atmosphäre, Quellen - (Aus)Wirkungen Messtechnik", Berlin, 2009.

[6] Gabrio, T., Strategie der Messung von Feinstaub in Innenräumen, Seminar "Nanopartikel in der Atmosphäre, Quellen - (Aus)Wirkungen - Messtechnik”, Berlin, 2009.

[7] McDermott, H.J., Air monitoring for toxic exposures ( $2^{\text {nd }}$ ed.), Real-time sampling methods for aerosols, John Wiley and Sons, Hoboken, New Jersey, 2004, p. 428.

[8] ISO 12103-1:1997, Road vehicles - Test dust for filter evaluation - Arizona test dust, 1997.

[9] DMT, Körnungskennlinie Dolomit 10 / Dolomit 90.

[10] Palas GmbH, RBG 1000 Particle Generator Manual, Karlsruhe, Germany, 2002.

[11] Behle, K., Standardization of False Alarm Rejection Capability Assessment, $14^{\text {th }}$ International Conference on Automatic Fire Detection, AUBE '09, Duisburg, Germany, September 8-10, 2009.

[12] EN54 Part 7, Fire detection and fire alarm systems - Part 7: Smoke detectors - Point detectors using scattered light, transmitted light or ionization; German version EN 54-7:2000 
[13] E+E Elektronik Gesellschaft m.b.H., Data sheet "Air Velocity- / Temperature Transmitter, Series EE70", Engerwitzdorf, Austria.

[14] LORENZ Meßgerätebau GmbH \& Co. KG, Obscuration-meter AML, Smoke-Tunnel-Version, 2009. 